\title{
Computational Study and Experimental Verification for Fluid Static Pressure in Centrifugal Pump Side Chamber
}

\author{
Sen Zhang ${ }^{1, \text { a }}$,Deke $\mathrm{Xi}^{2, \mathrm{~b}}$, Zhibin Liu ${ }^{3, \mathrm{c}}$, Zhidong Guo ${ }^{4, \mathrm{~d}}$ and Baofeng Chen ${ }^{5, \mathrm{e}}$ \\ ${ }^{1,2}$ Institute of Aeronautics, Northwestern Polytechnical University, Xi'an Shaanxi 710072, China \\ ${ }^{3,4,5}$ Shanxi Anrui Fan Electric Co., Ltd, Yuncheng Shanxi 044402, China

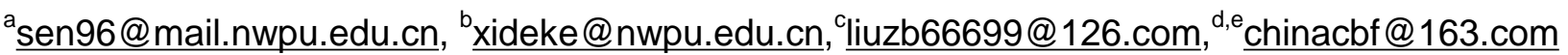

\begin{abstract}
Keywords: centrifugal pump; flow; pump side chamber; Navier-Stokes equations; pressure distribution; computation and verification

Abstract. A flow model was proposed to compute the fluid pressure in pump side chamber. Based on which the flow can be regarded as a secondary and axisymmetric viscous laminar flow, which is the superposition of circumferential shear flow and radial differential pressure one. These were described using N-S equations. By comparing orders of magnitude, the N-S equations were simplified. They were then integrated to obtain the analytical solutions. The correction coefficient of potential head was introduced to determine simplified computation model for fluid pressure inside chamber. By ignoring the influence of leakage, the final computation model was established. This research used the model to predict the pressure profiles of a centrifugal pump and the theoretical profile agrees well with the experimental one.
\end{abstract}

\section{Introduction}

In pump field, axial thrust balance theory is a very important topic. Scholars have summarized more than ten kinds of computational formulas to solve axial thrust. However, these formulas are mainly semi-empirical and semi-theoretical, by which axial thrust cannot be calculated exactly [1-8]. The solving of fluid pressure in pump chamber is a core research problem for the study of impeller shroud force, and it is the key in calculating axial thrust accurately. An assumption is that fluid in pump chamber has no leakage and rotates as a rigid body at half angular velocity of impeller. Based on the assumption, the fluid pressure in pump chamber is considered as a parabolic distribution along the radial direction. In fact, the assumption is not in accord with the real situation, which leads to a difference between calculation results and physical truth for axial thrust [9-12]. Gao et al. adopted an iterative method to solve axial thrust and indicated that in order to get an exact solution of impeller shroud force, Navier-Stokes equations (N-S equations) must be simplified by various methods for viscous flow in pump chamber [13]. A four-layer flow model for side chamber fluid was set up by Yang et al. Their research shows that most fluid rotates as a rigid body and its rotational angular speed is 0.48 times of the impeller one and not 0.5 times, as commonly considered [14]. With the development of computational techniques, the computational fluid dynamics (CFD) is widely used in flow analysis. Pump performance prediction and optimization design based on CFD has basically reached the level of practical engineering [15-17]. By numerical simulation, Shi et al. predicted the axial thrust for type 250QJ50 single-stage well-pump in main operation conditions and solved the axial thrust acting on twisted blade. The research enriches the solution of gross axial thrust [18]. In order to analyze the flow field structure of side chamber, Wang et al. adopted steady numerical simulation to the computational domains, which consists of side chamber, wear-ring clearance, volute and impeller channel. The results show that pump side chamber fluid pressure gradually decreases along the radial direction from inlet to inner [19]. In respect to the centrifugal pump with balance holes and double wear-rings, Liu et al. proposed a computational model to establish balance chamber fluid pressure and the reliability of the computational model was verified experimentally [20].

This work proposed a flow model for the incompressible viscous fluid and used N-S equations to describe it. By comparing orders of magnitude, the N-S equations were simplified. The analytical solutions were then obtained. According to the solution, a new theoretical formula was established to 
compute side chamber fluid pressure. Eventually, the type IS80-50-315 pump is measured to verify its practicability.

\section{Flow Model and Fundamental Assumptions}

The side chamber flow model is presented in Fig. 1. This model consists of impeller shroud, shaft and pump casing. In the figure, a cylindrical coordinate system $r-\theta-z$ is indicated, for which the r-axis is fixed on impeller shroud and the z-axis is fixed on pump casing, as shown in Fig. 1.

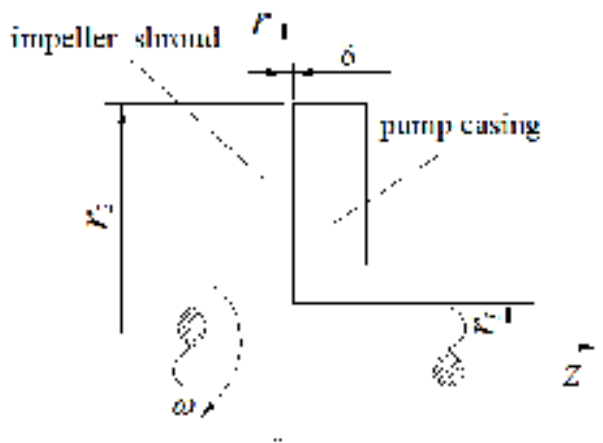

Fig. 1 The schematic of side chamber flow model

where $r_{2}$ stands for impeller outlet radius, $r_{1}$ represents radius of wear-ring, $\delta$ denotes the width of side chamber, $\omega$ is impeller rotational angular speed.

Some assumptions are made to determine the flow, which are as follows [21-26]:

(1) The fluid is incompressible and the flow is steady. Hence $\frac{\partial}{\partial t}=0$ and $\rho=c$ can be defined;

(2) The width of side chamber is small hence the axial velocity of fluid in side chamber is considered as zero, which can be written as $v_{z}=0$;

(3) The volute fluid is high pressure and flows into the side chamber inlet evenly so all flow parameters are not related with $\theta$. That is $\frac{\partial}{\partial \theta}=0$;

(4) For side chamber, the ratio of width and radial size is very small. Therefore, the fluid pressure is approximately regarded as changing only along the radial direction, which can be written as $\frac{\partial p}{\partial z}=0$.

On basis of the assumptions above, the flow in side chamber can be regarded as a secondary and axisymmetric viscous laminar one. It is the superposition of circumferential shear flow and radial differential pressure flow. The absolute flow is helical motion.

\section{Theoretical Computations for Liquid Pressure}

Approximate Analytical Solutions for N-S equations and Fluid Pressure Computation Model. The net flow induced by the leakage is inwards, thus the nominal radial velocity is also inwards. According to the model shown in Fig.1, the N-S equations for pump side chamber flow were simplified by ignoring the per unit mass force $[27,28]$, which can be written as

$$
\begin{aligned}
& -\frac{1}{\rho} \frac{\partial p}{\partial r}-v\left[\frac{\partial^{2} v_{r}}{\partial r^{2}}+\frac{\partial}{\partial r}\left(\frac{v_{r}}{r}\right)+\frac{\partial^{2} v_{r}}{\partial z^{2}}\right]=v_{r} \frac{\partial v_{r}}{\partial r}-\frac{v_{\theta}^{2}}{r} . \\
& v\left[\frac{\partial^{2} v_{\theta}}{\partial r^{2}}+\frac{\partial}{\partial r}\left(\frac{v_{\theta}}{r}\right)+\frac{\partial^{2} v_{\theta}}{\partial z^{2}}\right]=v_{r} \frac{\partial v_{\theta}}{\partial r}-\frac{v_{r} v_{\theta}}{r} .
\end{aligned}
$$




$$
-\frac{1}{\rho} \frac{\partial p}{\partial z}=0
$$

where $v_{r}$ stands for radial velocity of fluid in side chamber, $v_{\theta}$ is circumferential velocity of fluid in side chamber, $p$ fluid static pressure, $\rho$ denotes density of fluid, $v$ represents kinematic viscosity of liquid, which can be calculated byv $=\mu / \rho, \mu$ is dynamic viscosity of liquid.

The total differential equation for $p$ is expressed as

$$
\mathrm{d} p=\frac{\partial p}{\partial r} \mathrm{~d} r+\frac{\partial p}{\partial z} \mathrm{~d} z
$$

Substituting Eq.3 into Eq.4,

$$
\frac{\mathrm{d} p}{\mathrm{~d} r}=\frac{\partial p}{\partial r}
$$

For $\frac{\partial}{\partial \theta}=0$ and $v_{z}=0$, the continuity equation for incompressible fluid steady flow can be written as

$$
\frac{v_{r}}{r}+\frac{\partial v_{r}}{\partial r}=0
$$

Eq.6 is derived with $r$, which is written as

$$
\frac{\partial}{\partial r}\left(\frac{v_{r}}{r}\right)+\frac{\partial^{2} v_{r}}{\partial r^{2}}=0 .
$$

To simplify the N-S equations more, a method was adopted which compares the orders of magnitude for viscosity terms and inertial terms in $\mathrm{N}-\mathrm{S}$ equations, respectively. After the comparison, minor terms were neglected and the main terms retained.

For most centrifugal pump with a low specific speed, the impeller radius is very big in comparison to the width of side chamber, which can be written as $\delta=r_{2}$. From Fig.1, it can be determined that the value range of $\mathrm{r}$ is $r_{1} \leq r \leq r_{2}$ and the value range of $\mathrm{z}$ is $0 \leq z \leq \delta$ for $\mathrm{N}$-S equations. Therefore, $r$ and $r_{2}$ have the same orders of magnitude and so has $z$ and $\delta$, which are expressed as

$$
r \sim r_{2}, z \sim \delta
$$

When $r$ changes from $r_{1}$ to $r_{2}, \partial v_{r} / \partial r$ and $v_{r} / r_{2}$ have the same orders of magnitude which can be written as

$$
\frac{\partial v_{r}}{\partial r} \sim \frac{v_{r}}{r_{2}}
$$

Such typical theoretical treatments can be found in Refs. [29-31]. Similarly, we got some others as follows: 


$$
\frac{\partial v_{\theta}}{\partial r} \sim \frac{v_{\theta}}{r_{2}}, \quad \frac{\partial v_{\theta}}{\partial z} \sim \frac{v_{\theta}}{\delta}, \quad v_{r} \frac{\partial v_{r}}{\partial r} \sim \frac{v_{r}^{2}}{r_{2}}, \quad \frac{v_{\theta}^{2}}{r} \sim \frac{v_{\theta}^{2}}{r_{2}}, \quad v_{r} \frac{\partial v_{\theta}}{\partial r} \sim \frac{v_{r} v_{\theta}}{r_{2}}, \quad \frac{v_{r} v_{\theta}}{r} \sim \frac{v_{r} v_{\theta}}{r_{2}} .
$$

The orders of magnitude for partial differential terms were compared to the second order, which are expressed as

$$
\begin{aligned}
& \frac{\partial^{2} v_{\theta}}{\partial r^{2}}=\frac{\partial}{\partial r}\left(\frac{\partial v_{\theta}}{\partial r}\right) \sim \frac{v_{\theta}}{r_{2}^{2}} . \\
& \frac{\partial}{\partial r}\left(\frac{v_{\theta}}{r}\right) \sim \frac{v_{\theta}}{r_{2}^{2}} . \\
& \frac{\partial^{2} v_{\theta}}{\partial z^{2}} \sim \frac{\partial}{\partial z}\left(\frac{\partial v_{\theta}}{\partial z}\right) \sim \frac{v_{\theta}}{\delta^{2}} .
\end{aligned}
$$

As $\delta \ll r_{2}$, the orders of magnitude were compared for viscosity terms in Eq. 1 and Eq. 2 based on the results in Eq.8, Eq.9 and Eq.10. The relationships of viscosity terms can be expressed as

$$
v \frac{\partial^{2} v_{\theta}}{\partial r^{2}} \ll v \frac{\partial^{2} v_{\theta}}{\partial z^{2}}, v\left[\frac{\partial}{\partial r}\left(\frac{v_{\theta}}{r}\right)\right] \ll v \frac{\partial^{2} v_{\theta}}{\partial z^{2}}
$$

Then the orders of magnitude were compared for inertial terms in Eq. 1 and Eq.2, and because $v_{r} \ll v_{\theta}$, the expressions are expressed as

$$
v_{r} \frac{\partial v_{r}}{\partial r} \ll \frac{v_{\theta}^{2}}{r}, v_{r} \frac{\partial v_{\theta}}{\partial r} \ll \frac{v_{\theta}^{2}}{r}, \frac{v_{r} v_{\theta}}{r} \ll \frac{v_{\theta}^{2}}{r} .
$$

According to the results above, these five terms $v \frac{\partial^{2} v_{\theta}}{\partial r^{2}}$, $\mathrm{v}\left[\frac{\partial}{\partial r}\left(\frac{v_{\theta}}{r}\right)\right], v_{r} \frac{\partial v_{\theta}}{\partial r}, \frac{v_{r} v_{\theta}}{r}, v_{r} \frac{\partial v_{r}}{\partial r}$ can be neglected in Eq.1 and Eq.2. Eventually, after substituting Eq.7 into Eq.1, the N-S equations were simplified as follows:

$$
\begin{aligned}
& -\frac{1}{\rho} \frac{\partial p}{\partial r}-v \frac{\partial^{2} v_{r}}{\partial z^{2}}=-\frac{v_{\theta}^{2}}{r} . \\
& \frac{\partial^{2} v_{\theta}}{\partial z^{2}}=0 . \\
& -\frac{1}{\rho} \frac{\partial p}{\partial z}=0 .
\end{aligned}
$$

The circumferential velocity of fluid in pump side chamber was determined by integrating Eq.12 twice, which is written as

$$
v_{\theta}=c_{1} z+c_{2} .
$$

In order to solve the constants $c_{1}$ and $c_{2}$ in Eq.13, two boundary conditions were introduced. This was that the circumferential velocity of fluid in side chamber equals the one of impeller shroud or 
impeller when it is on the impeller shroud and the one on the pump casing equals zero, which can be expressed as $z=0, v_{\theta}=\omega r$ and $z=\delta, v_{\theta}=0$, respectively. These were then adopted into Eq.13.

$$
v_{\theta}=\omega r\left(1-\frac{z}{\delta}\right)
$$

where $\omega$ is determined by $\omega=\frac{2 \pi n}{60}, n$ represents the design revolving speed of pump, $\pi$ is a constant, $\pi=3.14$.

In order to obtain the expression of radial velocity of fluid in side chamber $v_{r}$, Eq.14 was substituted into Eq.11 and integrated twice. The expression is written as

$$
v_{r}=-\frac{1}{2 \mu} \frac{\mathrm{d} p}{\mathrm{~d} r} z^{2}+\frac{\rho \omega^{2} r}{\mu}\left(\frac{z^{4}}{12 \delta^{2}}-\frac{z^{3}}{3 \delta}+\frac{1}{2} z^{2}\right)+c_{3} z+c_{4} \text {. }
$$

Another two boundary conditions were introduced to solve $c_{3}$ and $c_{4}$ in Eq.15, that the radial velocity equals zero when it is on the impeller shroud and the one on the pump casing equals zero too, which can be expressed as $z=0, v_{r}=0$ and $z=\delta, v_{r}=0$, respectively. These were then adopted into Eq.15.

$$
v_{r}=\frac{1}{2 \mu} \frac{\mathrm{d} p}{\mathrm{~d} r}\left(\delta z-z^{2}\right)+\frac{\rho \omega^{2} r}{\mu}\left(\frac{z^{4}}{12 \delta^{2}}-\frac{z^{3}}{3 \delta}+\frac{1}{2} z^{2}-\frac{1}{4} \delta z\right) .
$$

The fluid leakage $q_{v}$ in pump chamber was determined by Eq.17 which is expressed as

$$
q_{v}=\int_{0}^{\delta} 2 \pi r v_{r} \mathrm{~d} z=\frac{\pi r \delta^{3}}{6 \mu} \frac{\mathrm{d} p}{\mathrm{~d} r}-\frac{\rho \pi \omega^{2} \delta^{3} r^{2}}{20 \mu}
$$

Rearranging Eq.17 derives

$$
\mathrm{d} p=\left(\frac{6 \mu q_{v}}{\pi \delta^{3} r}+\frac{3 \rho \omega^{2}}{10} r\right) \mathrm{d} r .
$$

Eq.18 indicates that the pressure gradient $\frac{\mathrm{d} p}{\mathrm{~d} r}$ is the function of leakage $q_{v}$ and radius $r$. Integrating Eq.18, allows the expression of fluid pressure, which is expressed as

$$
p=\frac{6 \mu q_{v}}{\pi \delta^{3}} \ln r+\frac{3}{20} \rho \omega^{2} r^{2}+c_{5} .
$$

In order to solve $c_{5}$, an assumption was made that the pressure at the impeller outlet equals the one at the side chamber inlet, which can be expressed as $r=r_{2}, p=p_{3}$. The assumption which acts as the boundary conditions was substituted into Eq.19. Eventually, by dividing Eq.19 with $\rho g$, the computation model was obtained.

$$
\frac{p}{\rho g}=\frac{p_{3}}{\rho g}-\frac{6 v q_{v}}{g \pi \delta^{3}} \ln \frac{r_{2}}{r}-1.2 \frac{u_{2}^{2}}{8 g}\left(1-\frac{r^{2}}{r_{2}^{2}}\right) .
$$


where $u_{2}$ stands for impeller tip speed.

Simplified Computation Model for Fluid Pressure. Zailun Liu et al. measured the fluid leakage rate through wear-ring using specialized equipment and obtained the fluid leakage rate profiles in terms of differential pressure between the front and rear of wear-ring [32]. A computational method for fluid leakage rate in side chamber was introduced by Zailun Liu et al. and by measuring the pressure in the front and rear of wear-ring the Head profiles in terms of fluid leakage rate were obtained [33]. Maoqing Chen simulated the operation condition of centrifugal pump by specialized equipment and measured the wear-ring fluid leakage rate [34]. These research indicate that the order of magnitude for the fluid leakage wear-ring is $10^{-3} \mathrm{~m}^{3} / \mathrm{h}$ under proper functioning. In general, the width of pump side chamber is more than five millimeters, which can be expressed as $\delta>5 \mathrm{~mm}$. Therefore, the term which contains $q_{v}$ in Eq.20 is so small that it can be neglected. Eq.20 was simplified as follows:

$$
\frac{p}{\rho g}=\frac{p_{3}}{\rho g}-1.2 \frac{u_{2}^{2}}{8 g}\left(1-\frac{r^{2}}{r_{2}^{2}}\right) .
$$

On the right hand side of Eq.21, $\frac{p_{1}}{\rho g}$ was added and subtracted at the same time, which is written as

$$
\frac{p}{\rho g}=\frac{p_{3}}{\rho g}-\frac{p_{1}}{\rho g}+\frac{p_{1}}{\rho g}-1.2 \frac{u_{2}^{2}}{8 g}\left(1-\frac{r^{2}}{r_{2}^{2}}\right) .
$$

where $p_{1}$ is the fluid static pressure at impeller inlet, which can be established by experiment.

The pump side chamber fluid is under the influence of its rotation which is driven by rotational impeller shroud and its radial flow caused by leakage, there is $p_{3}<p_{2}$. In order to compute the fluid pressure inside chamber inlet $p_{3}$, the correction coefficient of potential head $k$ was introduced. The definition is

$$
k=\frac{p_{3}-p_{2}}{p_{2}-p_{1}}
$$

where $0<k<1$, and it was suggested that $k=0.9: 0.95, p_{2}$ is fluid static pressure at impeller outlet.

According to the definition of potential head, Eq.23, was rearranged and expressed as

$$
\frac{p_{3}}{\rho g}-\frac{p_{1}}{\rho g}=k\left(\frac{p_{2}}{\rho g}-\frac{p_{1}}{\rho g}\right)=k H_{p} \text {. }
$$

Eventually, Eq.24 was substituted into Eq.22 and the simplified computation model was established, which is expressed as

$$
\frac{p}{\rho g}=k H_{p}-1.2 \frac{u_{2}^{2}}{8 g}\left(1-\frac{D^{2}}{D_{2}^{2}}\right)+\frac{p_{1}}{\rho g} .
$$

where the pump potential head $H_{p}$ was calculated by Eq.26, which is expressed as 


$$
H_{p}=H_{t}\left(1-\frac{g H_{t}}{2 u_{2}^{2}}\right)
$$

If the pre-circulation of flow in the inlet of impeller is ignored, there is $v_{u 1 \infty}=0$. For centrifugal pump with infinite blades, the theoretical head can be calculated using the following equation

$$
H_{t \infty}=\frac{u_{2}}{g}\left(u_{2}-v_{m 2} \cot \beta_{2}\right) \text {. }
$$

where $v_{m 2}$ is axial plane velocity of fluid at impeller outlet, $v_{m 2}=\frac{Q}{\pi D_{2} b_{2} \psi_{2} \eta_{v}}, Q$ pump flow rate, $D_{2}$ donates impeller outlet diameter, $b_{2}$ represents the outlet blade width, the blade blockage coefficient at impeller outlet $\psi_{2}=0.85: 0.95$, this work adopted $\psi_{2}=0.93, \eta_{v}$ is pump volumetric efficiency in design condition, which can be determined by $\eta_{v}=\frac{1}{1+0.68 n_{s}^{-2 / 3}}, n_{s}$ stands for specific speed of pump.

By method of mathematical statistics and regression analysis, Xijie He et al. obtained the equation for correction coefficient of theoretical head [35], which is written as

$$
\sigma=\frac{1.55 Z^{0.15}}{\sqrt[3]{\beta_{2}}}, Z \in(4,8), \beta_{2} \in(20,40)
$$

where $\sigma$ is correction coefficient of theoretical head, $Z$ number of blades.

Then, the pump theoretical head was established by

$$
H_{t}=\sigma H_{t_{\infty}} .
$$

The fluid pressure of pump inlet $p_{1}$ is known, hence Eq.25 is still unsolvable. However, it can be established by experiment. Ultimately, Eq.25 is solvable.

\section{Theoretical Computations and Experimental Verification}

Theoretical Computations for Fluid Pressure in Design. Type IS80-50-315 centrifugal pump was taken as the computational model whose design parameters are tabulated in Table. 1 and important geometries of the impeller are tabulated in Table.2.

Table. 1 The design parameters of type IS80-50-315 centrifugal pump

\begin{tabular}{cccc}
\hline$Q\left(\mathrm{~m}^{3} / \mathrm{h}\right)$ & $H(\mathrm{~m})$ & $n(\mathrm{r} / \mathrm{min})$ & $\eta(\%)$ \\
\hline 25 & 32 & 1450 & 52 \\
\hline
\end{tabular}

Table. 2 The important geometries of the impeller

\begin{tabular}{llc}
\hline & impeller outlet radius $\quad r_{2}(\mathrm{~mm})$ & 158 \\
\multirow{3}{*}{ Feature sizes } & outlet blade width $b_{2}(\mathrm{~mm})$ & 8 \\
& exit blade angle $\beta_{2}\left({ }^{\circ}\right)$ & 22 \\
& number of blade $Z$ & 5 \\
\hline
\end{tabular}

The measured pressure of pump inlet is zero, which can be expressed as $p_{1} / \rho g=0$. According to the parameters given in Table.1 and Table.2, adopting Eq.26 can be used to obtain the theoretical 
profiles of pressure inside chamber. When Eq.26 was solved, 0.95 was taken for $k$. The predicted profiles are shown in Fig.2.

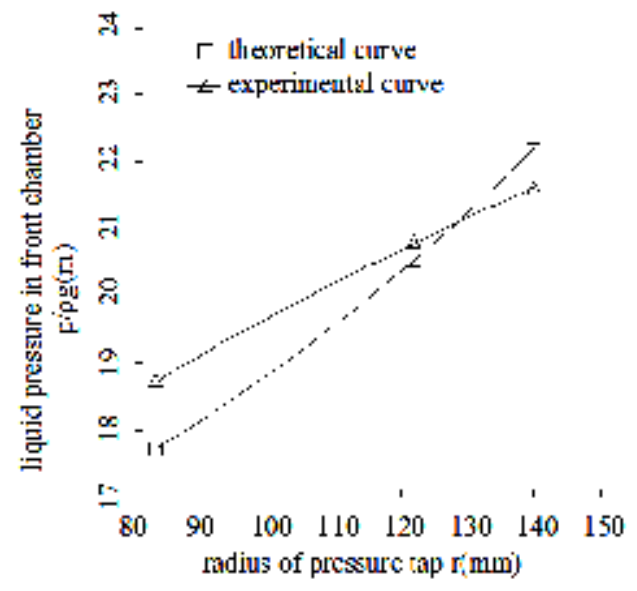

(a) Front side chamber

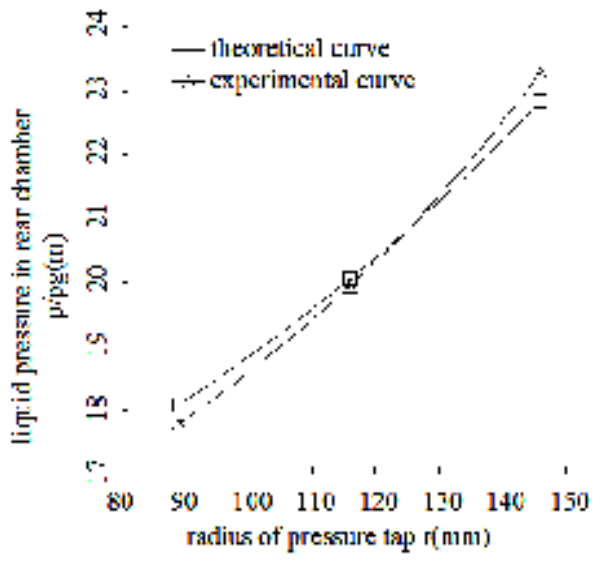

(b) Rear side chamber

Fig.2Comparison between the calculated and experimental profiles

Experiment Method and Results. The experiment was conducted on the closed test bench for floating impeller of centrifugal pump.

In order to measure the fluid pressure inside chamber, three pressure taps on the pump body were opened, which have radiuses of $140 \mathrm{~mm}, 122 \mathrm{~mm}$ and $83 \mathrm{~mm}$ respectively. There were three other pressure taps on the pump casing with radiuses of $146 \mathrm{~mm}, 116 \mathrm{~mm}$ and $89 \mathrm{~mm}$, respectively as shown in Fig.3.

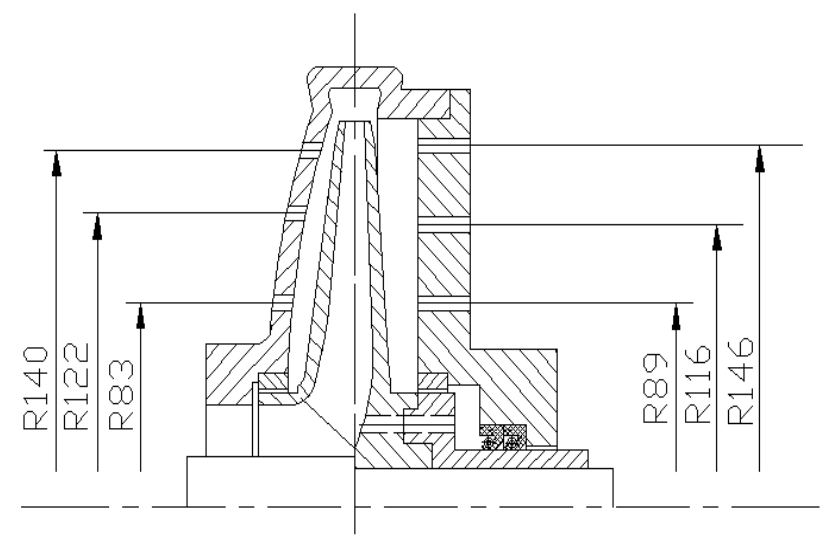

Fig.3 The positions of measuring taps

In design condition, when the measured flow rate of type IS80-50-315 centrifugal pump is $25.13 \mathrm{~m}^{3} / \mathrm{h}$, the measured head is $27.92 \mathrm{~m}$. Eq. 30 can convert measured fluid pressure into the one under design revolving speed.

$$
\frac{p}{\rho g}=\frac{p^{\prime}}{\rho g}\left(\frac{n}{n^{\prime}}\right)^{2} \text {. }
$$

where $\frac{p}{\rho g}$ is the fluid pressure head at design revolving speed and $\frac{p^{\prime}}{\rho g}$ is the fluid pressure head at experiment revolving one. 


\section{Discussion}

The experimental profiles were shown in Fig.2. In design, fluid pressure increases as radius increases, and the profile is almost a rising straight line. The theoretical profile agree well with the experimental one in rear chamber. However, the profiles do not agree as well in front chamber. Nearby front wear-ring inlet, the theoretical profile has a large difference with the experimental one. With a radius of $83 \mathrm{~mm}$, the difference between theoretical and experimental values is $1.01 \mathrm{~m}$. The experiment results also show that the average pressure in rear chamber nearly equals the one in front chamber and the deviation is $0.1 \mathrm{~m}$.

\section{Conclusions}

Based on the assumption of the flow model, the flow inside chamber can be regarded as a secondary and axisymmetric viscous laminar flow, which is the superposition of circumferential shear flow and radial differential pressure one. The N-S equations were simplified by comparing orders of magnitude. Then the N-S equations were integrated to obtain the analytical solutions. In order to determine the simplified computation model, the correction coefficient of potential head $k$ was introduced. When the fluid pressure in pump side chamber is predicted, the research suggest that $k$ takes $0.9: 0.95$. Eventually, by ignoring the influence of leakage, the final computation model was established. This was used to predict the pressure profiles for type IS80-50-315 centrifugal pump. The theoretical profile agrees well with the experimental observations. The result shows that the computation model can predict the pressure well for the pump. And when a centrifugal pump is designed, the model can predict pressure inside chamber well.

\section{References}

[1] Elsevier Science Ltd.: World Pumps, 393, pp. 34-37. (1999)

[2] Domm, U. and Zilling, H.: Preprint for the IAHR Symposium Pumps in Power Stations, Braunschweig, pp. J23-J34. (1966)

[3] Wenguang Li: ASME J. Fluids Eng., 135(1), p. 051201. (2013)

[4] Wenguang Li: ASME J. Fluids Eng., 134(1), p. 014501. (2012)

[5] Shnepp, V. B.: Chem. Petrol. Eng., 18(4), pp. 159-162. (1982)

[6] Xudan Ma, Dazhuan Wu, Leqin Wang: Transactions of the Chinese Society of Agricultural Engineering, 26(8), pp. 108-112. (2010)

[7] Iino, T., Sato, H., and Miyashiro, H.: ASME J. Fluids Eng., 102(1), pp. 64-69. (1980)

[8] Zuchao Zhu: Journal of Engineering thermo physics, 21(6), pp. 60-63. (2000) (In Chinese)

[9] Institute of Shenyang pumps and Academy of agricultural mechanization. Vane pump design manual, Beijing: China Machine Press. (1979)(In Chinese)

[10] Chengwei Ding. Theory and Hydraulic design of Centrifugal pump and axial flow pump, Beijing: China Machine Press. (1981) (In Chinese)

[11] Verba, A. and Sebestyen, G.: Preprint for the IAHR Symposium Pumps in Power Stations, Braunschweig, pp. J35-J42. (1966)

[12] Rennian Li, Hui Quan, Wei Han: Drainage and Irrigation Machinery, 30(5), pp. 257-531. (2012) (In Chinese)

[13]Hongli Gao, Jilong Yang, Li Ye, et al: Pump Technology, 2, pp. 8-12. (2002) (In Chinese)

[14]Junhu Yang, Chunlong Wang, Jinping Li: Transactions of the Chinese Society for Agricultural Machinery, 34(6), pp. 68-72. (2003) (In Chinese) 
[15]Weigang Lu, Jinfeng Zhang, Shouqi Yuan: China Mechanical Engineering, 18(17), pp. 2037-2040. (2007) (In Chinese)

[16]Qingshun Wei, Zailun Liu.: Proceedings of the CSEE, 31(14), pp. 103-107. (2011) (In Chinese)

[17]Fujun Wang, Yaojun Li, Wene Wang, et al: Drainage and Irrigation Machinery, 23(5), pp. 1-10. (2005) (In Chinese)

[18] Weidong Shi, Qifeng Li, Weigang Lu, et al: Transactions of the Chinese Society for Agricultural Machinery, 40(1), pp. 60-63. (2009) (In Chinese)

[19]Xiuyong Wang, Canxing Wang, Yibin Li: Transactions of the Chinese Society for Agricultural Machinery, 40(4), pp. 86-90. (2009) (In Chinese)

[20]Zailun Liu, Wei Dong, Nan Zhang, et al: Transactions of the Chinese Society of Agricultural Engineering, 29(20), pp. 54-59. (2013) (In Chinese)

[21] Junfeng Yan, Wei Chen: Journal of rocket propulsion, 3, pp. 20-25. (2007) (In Chinese)

[22] Suping Wen, Xiaowen Hu, Jun Wang: Journal of Engineering Thermo physics, 30(1), pp. 57-60. (2009) (In Chinese)

[23] Anker J E: Proceed-Dings of the Institution of Mechanical Engineers, 219(6), pp. 92-95. (2005)

[24]Dongyuan Pan, Tong Wang, Bin Zhang, et al: Chinese Journal of Hydrodynamics, 24(2), pp. 201-206. (2009) (In Chinese)

[25]Zirong Zhou, Xibing Li, Yingchun Liu: China Mechanical Engineering, 16(11), pp. 1009-1012. (2005) (In Chinese)

[26]Evgenev, S. S.:Chem. Petrol. Eng., 31(11), pp. 596-603. (1995)

[27]Fengyu Zhang, Xiaorui Cheng, Xiuyong Wang. Hydrodynamics, Beijing: China Water Power Press. (2012) (In Chinese)

[28] Yongquan Gu. Fluid Dynamic Seal, Beijing: China University of Petroleum Press. (1990) (In Chinese)

[29] Shuyan Liu, Weige Yan.: Journal of Beijing institute of technology, 7(2), pp. 113-119. (1988)

[30] Keqiang Zhao.: Journal of china ordnance archies of armored vehicles with engines. 38(2), pp. 38-44. (1990) (In Chinese)

[31] Wanyong Zhao, Liang Zhang, Zhen Wang.: Drainage and Irrigation Machinery, 26(4), pp. 14-17. (2008) (In Chinese)

[32]Zailun Liu, Rui He, Ying Fan: Society for Agricultural Machinery, 42(9), pp. 113-115. (2011) (In Chinese)

[33] Tanjun Liu, Wei Wang, Xiong Lu: Journa1 of Gansu University of Technology, 27(3), pp. 43-46. (2001) (In Chinese)

[34] Maoqing Chen, Chao Chen.: CPM, 21(1), pp. 23-26. (1993) (In Chinese)

[35]Xijie He, Xuesu Lao, et al: Pump Technology, 4, pp. 13-15. (1997) (In Chinese) 